\title{
Exogenous citrate impairs glucose tolerance and promotes visceral adipose tissue inflammation in mice
}

\author{
João G. B. Leandro, Jair M. Espindola-Netto, Maria Carolina F. Vianna, Lilian S. Gomez, \\ Thaina M. DeMaria, Monica M. Marinho-Carvalho, Patricia Zancan, Heitor A. Paula Neto and \\ Mauro Sola-Penna* \\ Departamento de Biotecnologia Farmacêutica, Faculdade de Farmácia, Universidade Federal do Rio de Janeiro, Rio de \\ Janeiro, RJ, 21941-903, Brazil
}

(Submitted 29 January 2015 - Final revision received 22 December 2015 - Accepted 23 December 2015-First published online 11 February 2016)

\section{Abstract}

Overweight and obesity have become epidemic worldwide and are linked to sedentary lifestyle and the consumption of processed foods and drinks. Citrate is a metabolite that plays central roles in carbohydrate and lipid metabolism. In addition, citrate is the additive most commonly used by the food industry, and therefore is highly consumed. Extracellular citrate can freely enter the cells via the constitutively expressed plasma membrane citrate transporter. Within the cytosol, citrate is readily metabolised by ATP-citrate lyase into acetyl-CoA - the metabolic precursor of endogenously produced lipids and cholesterol. We therefore hypothesised that the citrate ingested from processed foods and drinks could contribute to increased postprandial fat production and weight gain. To test our hypothesis, we administered citrate to mice through their drinking water with or without sucrose and monitored their weight gain and other metabolic parameters. Our results showed that mice receiving citrate or citrate + sucrose did not show increased weight gain or an increase in the weight of the liver, skeletal muscles or adipose tissues (AT). Moreover, the plasma lipid profiles (TAG, total cholesterol, LDL and HDL) were similar across all groups. However, the group receiving citrate + sucrose showed augmented fasting glycaemia, glucose intolerance and the expression of pro-inflammatory cytokines (TNF- $\alpha$, IL-1 $\beta$, IL- 6 and IL-10) in their AT. Therefore, our results suggest that citrate consumption contributes to increased AT inflammation and altered glucose metabolism, which is indicative of initial insulin resistance. Thus, citrate consumption could be a previously unknown causative agent for the complications associated with obesity.

Key words: Citrate: Obesity: Inflammation: Food additives: Insulin resistance: Diabetes

Overweight and obesity are currently a threat to public health. The number of overweight and obese individuals has more than doubled since 1980, and it is estimated that 39\% of the world population is overweight and $13 \%$ are obese ${ }^{(1,2)}$. This epidemic is largely attributed to the modern sedentary lifestyle and a 'westernised' diet, based on the consumption of caloric and processed foods and drinks. In that sense, much attention has been given to the contribution of the high fat and high sugar contents of modern food ${ }^{(3)}$. Meanwhile, the relative contribution of food additives has been largely ignored, possibly because of their negligible energy content and the assumption that food additives are metabolically inert.

Citrate is the most widely used additive in the food industry, as it is a very efficient food flavouring agent and preservative ${ }^{(4)}$. Citrate has been considered innocuous, despite the fact that it is a very important metabolite in virtually all cell types. Citrate is produced in the mitochondria in the first reaction of the Krebs cycle through the condensation of one acetyl-CoA and one oxaloacetate molecule. In a situation of excess energy (postprandially), citrate accumulates and is then exported to the cytoplasm. Cytoplasmic citrate is readily cleaved by ATP-citrate lyase (ACLY) to produce acetyl-CoA, which is a fundamental precursor for the endogenous production of lipids and cholesterol ${ }^{(4-7)}$.

Despite its importance as a food additive and as a metabolite, the metabolic fate of exogenous citrate is largely unknown. It is possible that exogenous citrate reaches the cytoplasm of various cells (most importantly, hepatocytes) and contributes, as a substrate, to the endogenous production of fatty acids and cholesterol. Therefore, citrate consumed in large excess from processed food or drinks could contribute to obesity and associated diseases.

The present study aimed to investigate the possible contribution of citrate supplementation to metabolic alterations related to Western diet-induced weight gain.

Abbreviations: AT, adipose tissues; GTT, glucose tolerance tests; ITT, insulin tolerance test.

* Corresponding author: Professor M. Sola-Penna, fax +55 2122801784 ext. 231, email maurosp@ufrj.br 


\section{Methods}

\section{Animals}

Throughout the study, we used male Swiss mice aged between 3 and 4 weeks at the start of treatment. Mice were randomly separated into four groups: a control group receiving water with chlorides ( $450 \mathrm{mg} / \mathrm{l} \mathrm{NaCl}$ and $100 \mathrm{mg} / \mathrm{l} \mathrm{KCl}$ ) (control group, CTRL); a group receiving a chloride solution with $15 \mathrm{~g} / \mathrm{l}$ sucrose (sucrose group, SUC); a group receiving a chloride solution with $9.25 \mathrm{~g} / 1$ sodium citrate (citrate group, CIT); and a group receiving sucrose and citrate (sucrose + citrate group, SUC-CIT). The concentrations of chlorides, sucrose and sodium citrate were those normally found in isotonic beverages. Mice had free access to regular mouse chow and water, except for an 8-h fasting period before we collected blood samples for fasting glycaemia, lipid profile and glucose tolerance tests (GTT). Weight gain in addition to food and water consumption was periodically monitored. All animal handling procedures were in accordance with our Institutional Guidelines, and the protocols used were approved by the Institutional Committee for the Use of Animals (protocol code FARMACIA08-05/16).

\section{Sample harvesting}

After $75 \mathrm{~d}$ of treatment, food was removed from the cage, and mice had free access to drinking water only. After food withdrawal of $8 \mathrm{~h}$, mice were killed, and blood and tissue samples were collected. The blood samples were centrifuged (10000 $\boldsymbol{g}$ for $15 \mathrm{~min}$ at $4^{\circ} \mathrm{C}$ ), and plasma was collected and stored at $-80^{\circ} \mathrm{C}$ until processing. Adipose tissues (AT) samples were snap-frozen and stored at $-80^{\circ} \mathrm{C}$ until processing. A sample of liver tissue was transferred to a container with $1 \%$ formol solution in PBS for later histological processing.

\section{Glucose tolerance test and insulin tolerance test}

Mice were deprived of food for $8 \mathrm{~h}$ for GTT or $4 \mathrm{~h}$ for insulin tolerance test (ITT). A small blood sample was harvested from the tail vein to determine baseline (fasting) glycaemia. Mice were then injected intraperitoneally with a $2 \mathrm{~g} / \mathrm{kg}$ glucose solution (GTT) or $0.5 \mathrm{U} / \mathrm{kg}$ insulin (Humalin ${ }^{\circledR} \mathrm{R}$; Eli Lilly), and glycaemia was monitored at 15, 30, 60 and 120 min after injection.

\section{Quantification of plasma lipid fractions}

Quantification of TAG, total cholesterol, LDL and HDL were carried out using plasma collected from mice, using commercially available kits (Doles) and by following the manufacturer's instructions.

\section{Histological preparation and image analysis}

Liver samples were embedded in paraffin and cut into $10-\mu \mathrm{m}$ sections. Samples were haematoxylin-eosin stained and analysed using an optical light microscope under 400x magnification. Images from at least five random fields from each sample were acquired and analysed. Image analysis was carried out using ImageJ software for Windows. We used the built-in particle counter to measure the area fraction occupied by small round particles ( $<300$ pixels), which corresponded to the small lipid droplets inside hepatocytes.

\section{Quantification of inflammatory markers}

Tissues were homogenised in a $0 \cdot 1 \%$ Triton X-100 solution in PBS. Homogenates were transferred to Eppendorf tubes and centrifuged $\left(10000 \boldsymbol{g}\right.$ for $15 \mathrm{~min}$ at $\left.4^{\circ} \mathrm{C}\right)$. The supernatant was collected, transferred to a new tube and stored at $-80^{\circ} \mathrm{C}$ until use. Concentrations of TNF- $\alpha$, IL- $1 \beta$, IL- 6 and IL-10 were quantified in tissue homogenates using commercially available kits following the manufacturer's instruction (Peprotech).

\section{Statistical analysis}

All experiments were performed at least twice with at least eight mice per group. All data are presented as means with their standard errors derived from all experiments. Statistical analysis was carried out using GraphPad Prism software for Mac. For multiple comparisons, we used a one-way ANOVA test with Tukey's posttest, and $P<0.05$ was considered to be statistically significant.

\section{Results}

\section{Effect of citrate supplementation on weight gain}

We first tested whether citrate consumption would affect weight gain in mice fed a standard chow. As most citrate can be derived from processed drinks, we also provided a group of mice a combination of citrate and sucrose. As shown in Fig. 1(a), mice receiving citrate or citrate + sucrose did not show a significant increase in body weight after $75 \mathrm{~d}$. Moreover, all groups had a similar total energy intake (Fig. 1(b)) and consumed similar amounts of water and food (Fig. 1(c) and (d), respectively). These results suggest that citrate intake does not impact weight gain after $75 \mathrm{~d}$.

\section{Effect of citrate supplementation on muscles, adipose tissues and liver}

We then tested whether citrate consumption would increase body fat content or would impact tissue weight. As shown in Fig. 2, mice from all groups had similar weight for skeletal muscles (quadriceps, Fig. 2(a)); total fat (Fig. 2(b)), perigonadal AT (Fig. 2(c)), mesenteric AT (Fig. 2(d)), retroperitoneal AT (Fig. 2(e)) and inguinal AT (Fig. 2(f)); and liver (Fig. 2(g)). Moreover, the lipid content of the liver, as quantified in haematoxylin-eosin-stained liver slides (data not shown) by measuring the area of lipid droplets inside the cytoplasm of hepatocytes, was similar among the groups (Fig. 2(h)). Together, these results show that citrate supplementation does not affect fat storage in the body after $75 \mathrm{~d}$.

\section{Effect of citrate on lipid levels}

As citrate is a precursor metabolite for lipid and cholesterol synthesis, we investigated whether citrate supplementation 

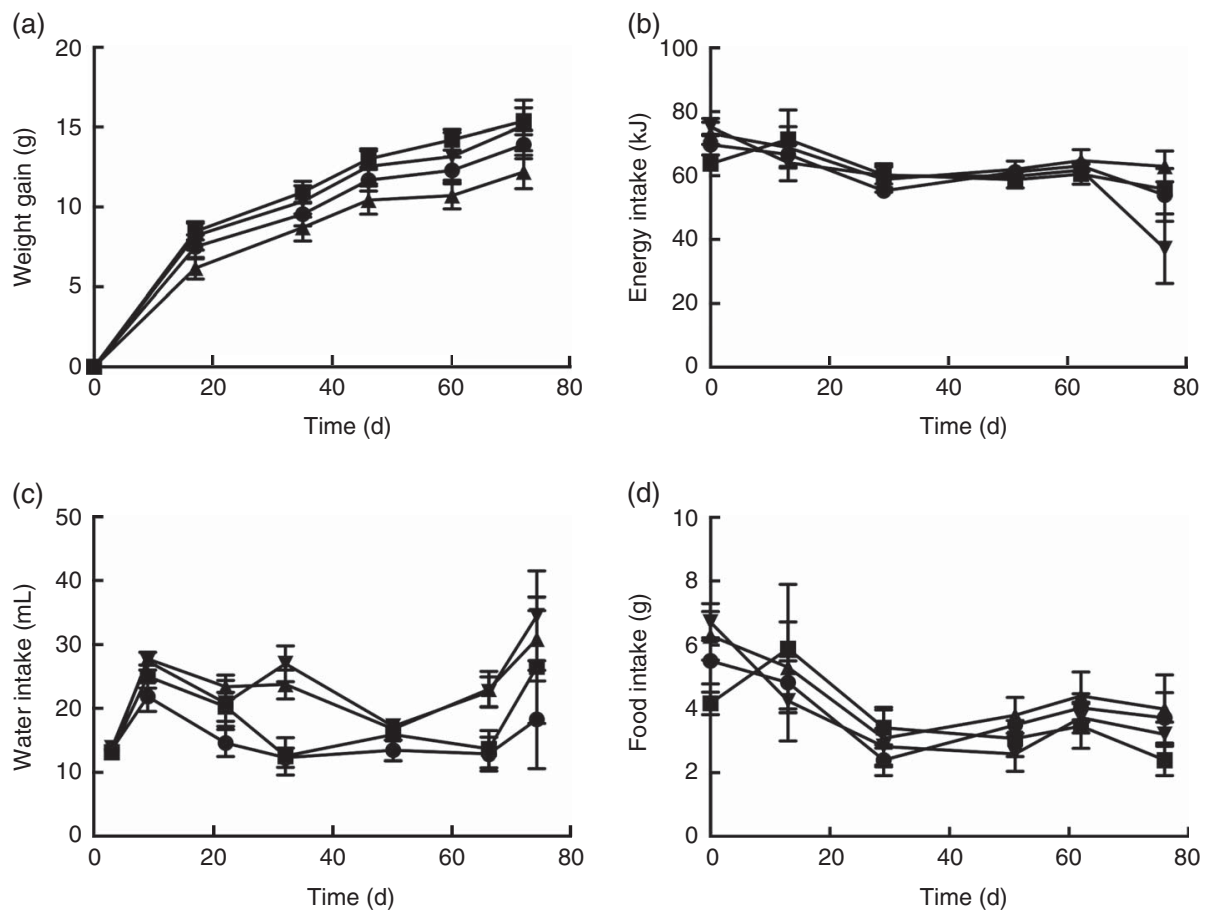

Fig. 1. Effects of citrate supplementation on weight gain. Total body weight (a), total energy intake (b), water intake (c) and food intake (d) of mice receiving chlorides (CTRL, $\mathbf{O})$, sucrose (SUC, $\square$ ), citrate (CIT, $\mathbf{\Delta}$ ) or sucrose and citrate (SUC $+\mathrm{CIT}, \boldsymbol{\nabla})$ for $75 \mathrm{~d}$. Body weight, food and water consumption were regularly monitored, and total energy intake was calculated based on the intake of food and water. Data are presented as means with their standard errors of three independent experiments with at least five mice in each group per experiment. Data were analysed by one-way ANOVA followed by Tukey's post-test, and $P<0.05$ was considered to be statistically significant.



(b)
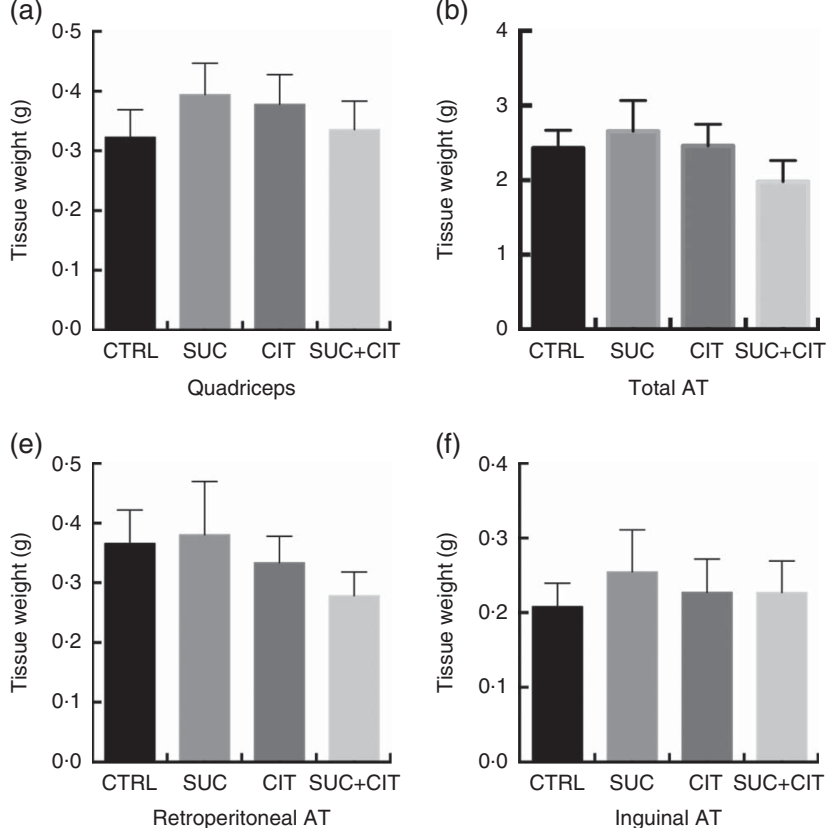

(f)

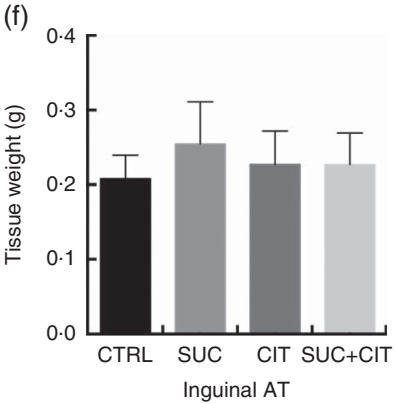

(c)

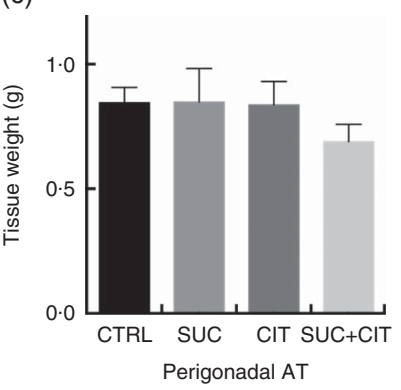

(g)

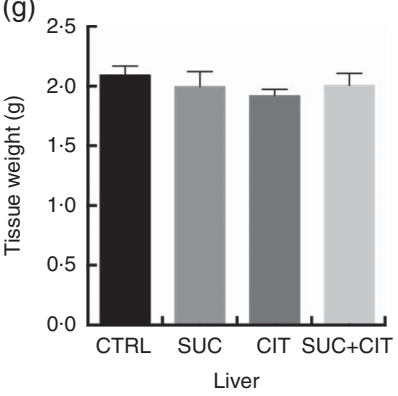

(d)



(h)

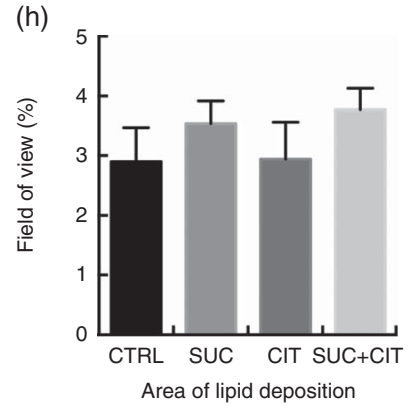

Fig. 2. Effects of citrate supplementation on adipose tissue (AT) and liver. The relative tissue weights to the total body weight were calculated for quadriceps (a), total fat (b), perigonadal AT (c), mesenteric AT (d), retroperitoneal AT (e), inguinal AT (f) and liver (g) from tissue samples of mice receiving chlorides (CTRL, $\square$ ), sucrose (SUC, ), citrate (CIT, ) or sucrose and citrate (SUC + CIT, ) for $75 \mathrm{~d}$. The panel $(\mathrm{h})$ presents the area fraction corresponding to intracellular lipid droplets observed in the histological analysis of the liver harvest from mice submitted to the different treatments as a parameter of ectopic fat deposition within hepatocytes. Data are presented as means with their standard errors of three independent experiments with at least five mice in each group per experiment represented by vertical bars. Data were analysed by one-way ANOVA followed by Tukey's post-test, and $P<0.05$ was considered to be statistically significant. 
could affect the lipid profiles of mice. We evaluated circulating TAG (Fig. 3(a)), total cholesterol (Fig. 3(b)), LDL (Fig. 3(c)) and HDL (Fig. 3(d)) in the plasma, and none of the treatments altered plasma lipid levels in mice after $75 \mathrm{~d}$.

\section{Effect of citrate supplementation on glucose homoeostasis}

We next evaluated fasting glucose levels, GTT and ITT in our experimental groups. Mice that received citrate and sucrose showed significantly increased fasting glucose levels (Fig. 4(a)). Accordingly, this group of mice also showed impaired GTT, with a higher glycaemic peak and slower glucose clearance following a single glucose infusion (Fig. 4(b)). This result was confirmed when we evaluated the area under the GTT curve, where this parameter was significantly higher for the group that received citrate and sucrose, when compared with the control (Fig. 4(c)). However, the ITT curves were similar across all the groups (Fig. 4(d)), which was confirmed by calculating the area under the ITT curves (Fig. 4(d)).

\section{Effect of citrate supplementation on adipose tissue inflammation}

Localised, chronic and low-grade AT inflammation underlies the development of insulin resistance in various animal models and in humans. We evaluated the levels of four pro-inflammatory cytokines that are normally increased in insulin-resistant models: TNF- $\alpha$ (Fig. 5(a)), IL-1 $\beta$ (Fig. 5(b)), IL-6 (Fig. 5(c)) and IL-10 (Fig. 5(d)). The levels of these four cytokines were significantly higher in the group that received citrate together with sucrose (Fig. 5). The cytokine levels in the AT of mice that
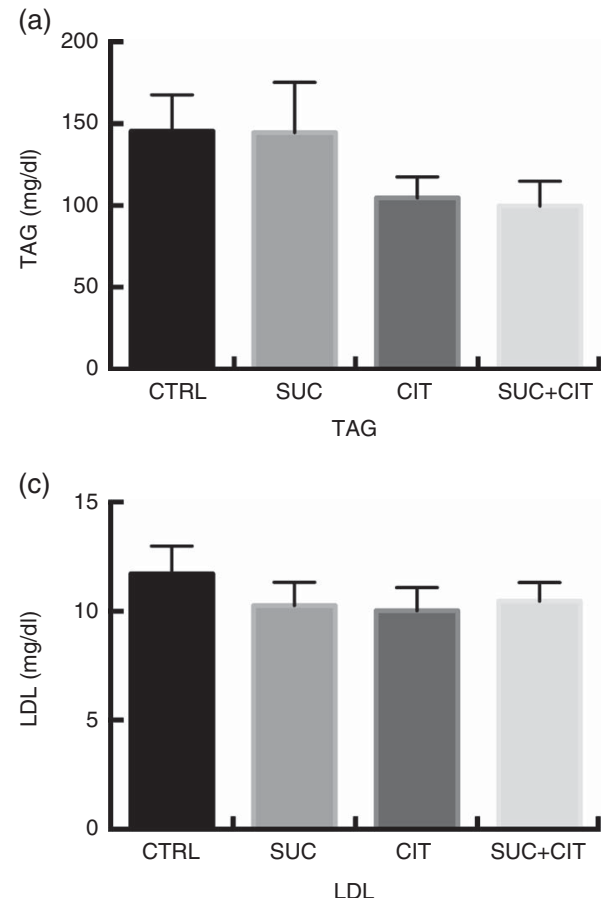

received either citrate or sucrose alone were slightly but not significantly increased relative to controls.

\section{Discussion}

Citrate is a very common additive used widely by the food industry. It is assumed that citrate supplementation has no major impact on the energy content of food ${ }^{(4)}$; however, in addition to being a food additive, citrate is a metabolite and, more importantly, is a common precursor for lipid and cholesterol synthesis. It is therefore reasonable to speculate that exogenous citrate could represent a relevant contributor to increased postprandial lipid synthesis and fat deposition. In fact, the consumption of processed foods and drinks with high energy and citrate content is a major contributor to the obesity epidemic ${ }^{(3)}$.

In the present study, we carried out a series of experiments to test the effects of citrate supplementation on various metabolic parameters in mice. Our results show that mice receiving citrate alone or in association with sucrose (as is found in nearly all processed drinks) did not result in a major increase in total body weight or in body fat content. Moreover, these mice presented no signs of ectopic lipid deposition or dyslipidaemia. Together, these results suggested that exogenous citrate might not function as a relevant substrate for endogenous lipid and cholesterol production, and therefore might not significantly contribute to fat deposition.

The relative short-term of our analyses ( $75 \mathrm{~d}$ ) could account for the lack of significant results. Studies usually report protocols of high-fat diet regimens using time courses of 8-12 weeks (56-84d) to observe effects on lipid deposition and weight gain $^{(8-10)}$. Furthermore, we have not used a high-fat diet but rather citrate supplementation, which despite the fact that it did

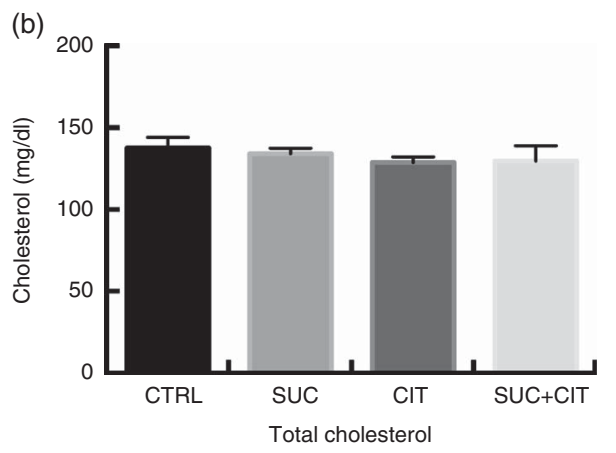

(d)

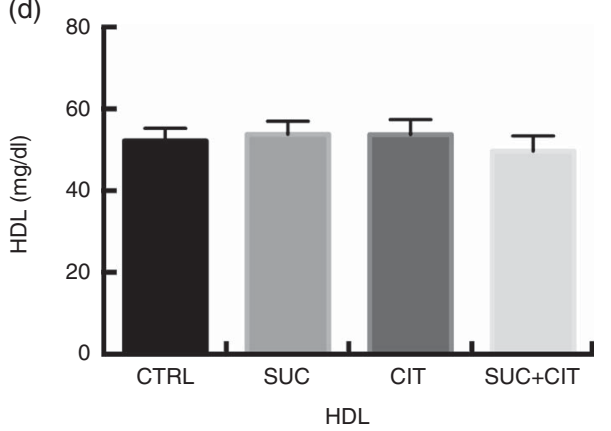

Fig. 3. Effects of citrate on cholesterol levels. Levels of TAG (a), total cholesterol (b) and LDL (c) and HDL (d) fractions were determined in plasma samples from mice receiving chlorides (CTRL, $\square$ ), sucrose (SUC, $\square$ ), citrate (CIT, $\square$ ) or sucrose and citrate (SUC+CIT, $\square$ ) for $75 \mathrm{~d}$. Data are presented as means with their standard errors of three independent experiments with at least five mice in each group per experiment represented by vertical bars. Data were analysed by one-way ANOVA followed by Tukey's posttest, and $P<0.05$ was considered to be statistically significant. To convert TAG to mmol//, multiply by 0.0113 . To convert cholesterol, LDL and $\mathrm{HDL}$ to $\mathrm{mmol} / \mathrm{l}$, multiply by 0.0259 . 
(a)

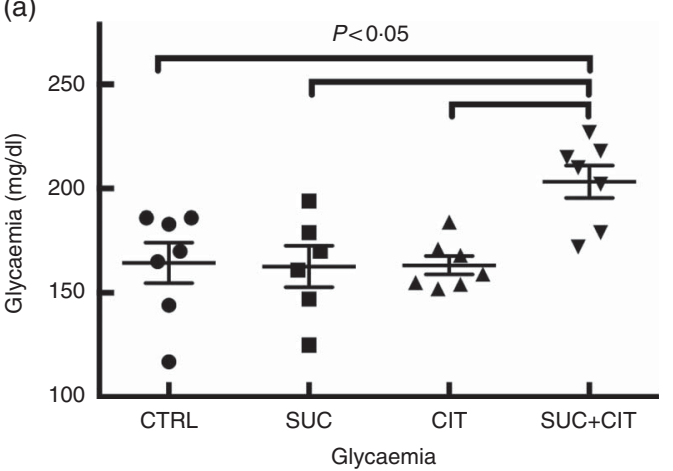

(b)

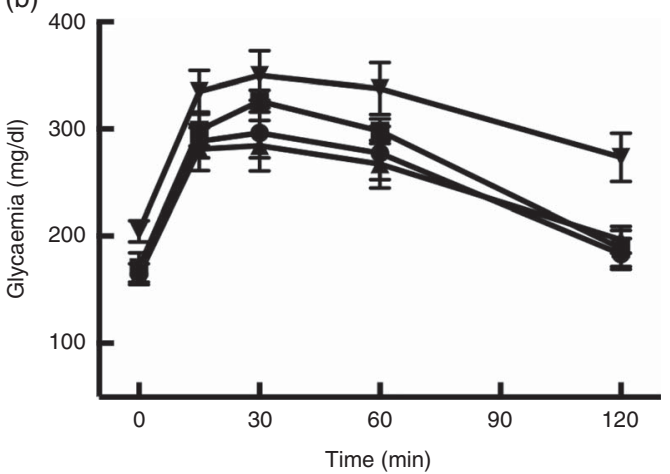

GTT

(d)

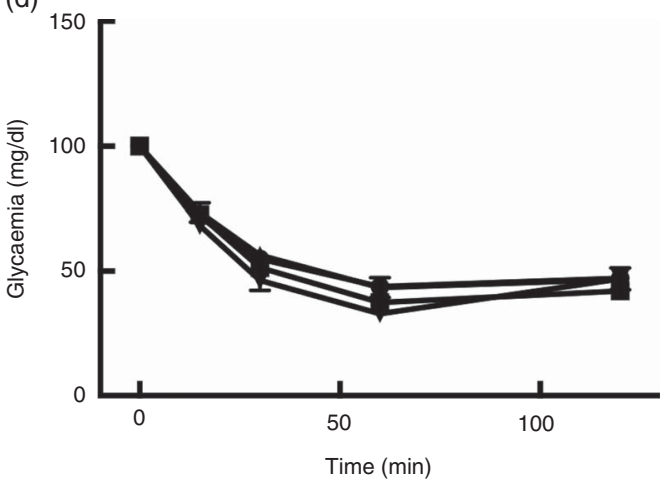

ITT

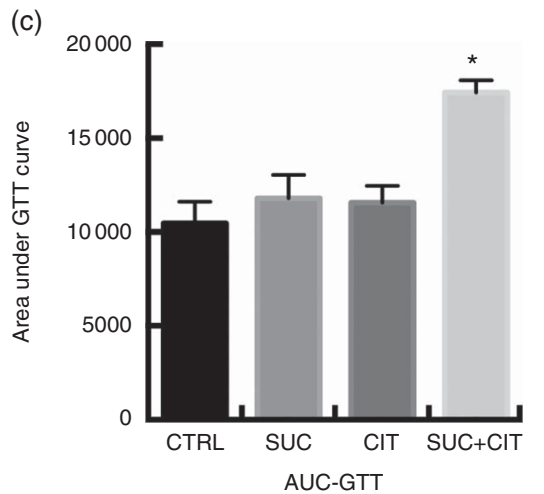

(e)

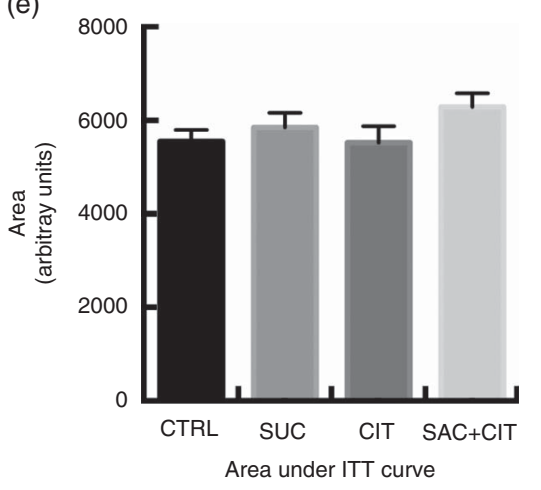

Fig. 4. Effects of citrate supplementation on glucose homoeostasis. Fasting glucose levels (a), glucose tolerance test (GTT) (b and c) and insulin tolerance test (ITT) ( $d$ and e) in mice receiving chlorides (CTRL), sucrose (SUC), citrate (CIT) or sucrose and citrate (SUC + CIT) for $75 \mathrm{~d}$. The AUC in the GTT graph (b) was determined and plotted (c). The AUC in the ITT graph (d) was determined and plotted (e). Data are presented as means with their standard errors of three independent experiments with at least five mice in each group per experiment. Data were analysed by one-way ANOVA followed by Tukey's post-test, and $P<0.05$ was considered to be statistically significant. * Significant difference relative to control group. a, b, d: , CTRL; $\boldsymbol{\square}$, SUC; $\boldsymbol{\Delta}$, CIT; $\boldsymbol{\nabla}$, SUC+CIT. c and d: $\boldsymbol{\square}$, CTRL; $\square$, SUC; $\square$, CIT; $\square$, SUC+CIT. To convert glycaemia to $\mathrm{mmol} / \mathrm{l}$, multiply by 0.0555 .

not alter lipid deposition promoted significant effects on other metabolic markers.

Interestingly, we found significant effects of citrate consumption when we analysed parameters of glucose homoeostasis. In mice receiving citrate and sucrose, we found a significantly increased fasting glycaemia, which was suggestive of insulin resistance. This was further confirmed by GTT, in which citrate + sucrose-treated mice presented glucose intolerance. However, we did not observe any alterations in the ITT, revealing that insulin response of the entire body was not yet compromised. This result indicates that what was observed was an initial state of glucose intolerance, which was confirmed by the inflammatory markers, as described below.

In search for an underlying mechanism, we evaluated the inflammatory response in AT. We found that concentrations of TNF- $\alpha$, IL- $1 \beta$, IL- 6 and IL- 10 were increased in AT of the animals that received citrate and sucrose. It is well documented that lowgrade AT inflammation correlates with insulin resistance in both humans and model organisms ${ }^{(11-13)}$. Our results suggest that citrate might make AT more prone to developing inflammation. 
(a)



(c)



(b)

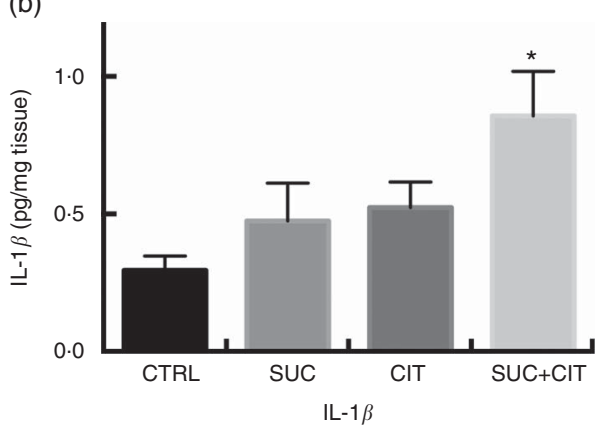

(d)



Fig. 5. Effects of citrate supplementation on adipose tissue inflammation. TNF- $a$ (a), IL-1 $\beta$ (b), IL-6 (c) and IL-10 (d) levels were quantified by ELISA in tissue homogenates from epididymal fat samples harvested from mice receiving chlorides (CTRL, $\square$ ), sucrose (SUC, $\square$ ), citrate (CIT, $\square$ ) or sucrose and citrate $(\mathrm{SUC}+\mathrm{CIT}, \quad)$ for $75 \mathrm{~d}$. Data are presented as means with their standard errors of three independent experiments with at least five mice in each group per experiment represented by vertical bars. Data were analysed by one-way ANOVA followed by Tukey's post-test, and $P<0.05$ was considered to be statistically significant. * Significant difference relative to control group.

It has already been shown that citrate metabolism contributes to the pro-inflammatory activity of macrophages in response to lipopolysaccharide ${ }^{(14,15)}$. In this context, ACLY plays a major role, highlighting the fact that cytosolic citrate is responsible for this inflammatory pattern ${ }^{(14,15)}$. Moreover, the acetylation and inhibition of the mitochondrial citrate transporter strongly diminishes the lipopolysaccharide-induced inflammatory response ${ }^{(16)}$. These data are physiologically relevant because the natural source of citrate is the mitochondria, where it is intracellularly produced. However, exogenous citrate also augments the lipopolysaccharide-activated monocyte inflammatory response ${ }^{(17)}$. This fact supports the hypothesis that citrate consumed in food and drinks could promote inflammation, as described above. Furthermore, hydroxycitrate, a known inhibitor of ACLY, attenuates diet-induced weight gain, lipid accumulation and AT inflammation ${ }^{(18,19)}$. Together, these studies corroborate our data and our conclusion that consumed citrate contributes to AT inflammation and ultimately to fasting hyperglycaemia. Although our results do not support the hypothesis that animals experience insulin resistance, the observed adipose inflammation and fasting hyperglycaemia suggest that they are in an initial glucose intolerance state, which over the medium- to long-term could lead to insulin resistance and diabetes.

The fact that the alterations we described depended on the association of citrate and sucrose does not represent a limitation of our hypothesis. Citrate is more often consumed together with carbohydrate sources such as the sucrose present in processed drinks and foods. Therefore, it is likely that the effects we described occur during human consumption of industrialised goods. Nonetheless, additional studies should be performed to address the synergism between citrate and sucrose that promoted the effects reported.

In summary, the results presented here support the hypothesis that citrate, far from being an inert food additive, could contribute to the establishment of local, low-grade inflammation. As this effect is closely related to insulin resistance, its use in the food industry should be revisited.

\section{Acknowledgements}

The authors thank Marcelo T. Bozza for help with the quantification of the cytokines, Philippe St-Pierre for discussion concerning the animal protocols and statistical analyses and Patricia L. Mitchell for discussion of the results and English usage.

This study was supported by grants from CAPES, CNPq and FAPERJ.

J. G. B. L. performed most of the experiments and helped in analysing the results; J. M. E.-N., M. C. F. V., L. S. G., T. M. D. M., P. Z. and M. M. M.-C. contributed to the experiments; P. Z., H. A. P. N. and M. S.-P. supervised the research and helped with discussion and data analysis; H. A. P. N. performed the ELISA; and H. A. P. N. and M. S.-P. wrote the paper.

The authors declare that there are no conflicts of interest.

\section{References}

1. Johnson AR \& Makowski L (2015) Nutrition and metabolic correlates of obesity and inflammation: clinical considerations. J Nutr 145, 1131S-1136S. 
2. Kelly T, Yang W, Chen CS, et al. (2008) Global burden of obesity in 2005 and projections to 2030. Int J Obes (Lond) 32, 1431-1437.

3. Popkin BM, Adair LS \& Ng SW (2012) Global nutrition transition and the pandemic of obesity in developing countries. Nutr Rev 70, 3-21.

4. Evans G, de Challemaison B \& Cox DN (2010) Consumers' ratings of the natural and unnatural qualities of foods. Appetite 54, 557-563.

5. Chu KY, Lin Y, Hendel A, et al. (2010) ATP-citrate lyase reduction mediates palmitate-induced apoptosis in pancreatic beta cells. J Biol Chem 285, 32606-32615.

6. Gameiro PA, Yang J, Metelo AM, et al. (2013) In vivo HIF-mediated reductive carboxylation is regulated by citrate levels and sensitizes VHL-deficient cells to glutamine deprivation. Cell Metab 17, 372-385.

7. Chypre M, Zaidi N \& Smans K (2012) ATP-citrate lyase: a mini-review. Biochem Biophys Res Commun 422, 1-4.

8. Ojo OO, Srinivasan DK, Owolabi BO, et al. (2015) MagaininAM2 improves glucose homeostasis and beta cell function in high-fat fed mice. Biochim Biophys Acta 1850, 80-87.

9. Ferreira AV, Menezes-Garcia Z, Viana JB, et al. (2014) Distinct metabolic pathways trigger adipocyte fat accumulation induced by high-carbohydrate and high-fat diets. Nutrition 30, 1138-1143.

10. Carvalho BM, Guadagnini D, Tsukumo DM, et al. (2012) Modulation of gut microbiota by antibiotics improves insulin signalling in high-fat fed mice. Diabetologia 55, 2823-2834.

11. Kredel LI \& Siegmund B (2014) Adipose-tissue and intestinal inflammation - visceral obesity and creeping fat. Front Immunol 5, 462
12. Esser N, Legrand-Poels S, Piette J, et al. (2014) Inflammation as a link between obesity, metabolic syndrome and type 2 diabetes. Diabetes Res Clin Pract 105, 141-150.

13. Labonte AC, Tosello-Trampont AC \& Hahn YS (2014) The role of macrophage polarization in infectious and inflammatory diseases. Mol Cells 37, 275-285.

14. Iacobazzi V \& Infantino V (2014) Citrate - new functions for an old metabolite. Biol Chem 395, 387-399.

15. Infantino V, Iacobazzi V, Palmieri F, et al. (2013) ATP-citrate lyase is essential for macrophage inflammatory response. Biochem Biophys Res Commun 440, 105-111.

16. Palmieri EM, Spera I, Menga A, et al. (2015) Acetylation of human mitochondrial citrate carrier modulates mitochondrial citrate/malate exchange activity to sustain NADPH production during macrophage activation. Biochim Biophys Acta 1847, 729-738.

17. Ashbrook MJ, McDonough KL, Pituch JJ, et al. (2015) Citrate modulates lipopolysaccharide-induced monocyte inflammatory responses. Clin Exp Immunol 180, 520-530.

18. Shara M, Ohia SE, Yasmin T, et al. (2003) Dose- and timedependent effects of a novel (-)-hydroxycitric acid extract on body weight, hepatic and testicular lipid peroxidation, DNA fragmentation and histopathological data over a period of 90 days. Mol Cell Biochem 254, 339-346.

19. Asghar M, Monjok E, Kouamou G, et al. (2007) Super CitriMax (HCA-SX) attenuates increases in oxidative stress, inflammation, insulin resistance, and body weight in developing obese Zucker rats. Mol Cell Biochem 304, 93-99. 